\title{
NATIONAL SPATIAL DATA INFRASTRUCTURE (NSDI) OF BANGLADESH- DEVELOPMENT, PROGRESS AND WAY FORWARD
}

\author{
Md. Mostafizur Rahman ${ }^{1,3 *}$, György Szabó ${ }^{2}$ \\ ${ }^{1}$ Department of Photogrammetry and Geoinformatics, Faculty of Civil Engineering, Budapest University of \\ Technology and Economics, Hungary - mostafizur.rahman@epito.bme.hu \\ ${ }^{2}$ Department of Photogrammetry and Geoinformatics, Faculty of Civil Engineering, Budapest University of \\ Technology and Economics, Hungary - szabo.gyorgy@epito.bme.hu \\ ${ }^{3}$ Department of Urban \& Regional Planning, Rajshahi University of Engineering \& Technology, \\ Bangladesh - mostafizur@urp.ruet.ac.bd
}

Commission IV, WG IV/6

KEY WORDS: National Spatial Data Infrastructure, Digital Bangladesh, GeoDASH, Geospatial Data, Geoportal, SDGs,

\begin{abstract}
:
National Spatial Data Infrastructure (NSDI) refers to a framework of policies, institutional arrangements, technologies, data, and people that enables the sharing and effective usage of geographic information. This paper aims to present the development and current progress of NSDI in Bangladesh. In preparing this paper, we have reviewed several papers, documents and articles related to NSDI development in Bangladesh. We have also explored different spatial data sharing platforms of related agencies and departments. This study finds that, in last decade, there is substantial increase in the production and use of geospatial data in Bangladesh. However, due to the absence of planning and centralized spatial data infrastructure (SDI) system, there are duplication of efforts among different organizations in respect of spatial data production leading to wastage of time and financial resources. There is also problem of maintaining same metadata standard among different organisations creating difficulties in building and sharing spatial data. Against this background, in 2016, Bangladesh government has decided to develop NSDI. As there are some organizations already have developed geoportal so it is decided that existing platform will be used as NSDI initially. Based on this decision GeoDASH platform is being used at present. However, there are many challenges and much to develop the platform. Development of NSDI in Bangladesh is still under process and there are some challenges also. Overcoming the problems and challenges, full- fledged development and operation of NSDI is expected to support the achievement of SDGs in Bangladesh.
\end{abstract}

\section{INTRODUCTION}

Spatial Data Infrastructure (SDI) is a system of gathering, sharing and disseminating spatially refenced data and information in a coordinated manner with technological support in order to ease policy formulation and decision making to meet the societal needs in local, national and global scale (NoguerasIso, Zarazaga-Soria and Muro-Medrano, 2005; Pashova and Bandrova, 2017). The SDI concept is continuously becoming very popular within the broad spectrum of socio-economic sphere of government for its visible benefits regarding policy making and development plan formulation. The development of SDI is contributing a lot to the decision maker by providing them spatially referenced data and information for effective resource allocation, good governance, socio-economic growth and sustainable development (Dale and Allan, 2004). When SDI system is developed and maintained nationally, it becomes National Spatial Data Infrastructure. The concept of "National Spatial Data Infrastructure (NSDI)" was first evolved in US when they published the Presidential Documents as the Federal Register on April 13, 1994 (Clinton, 1994). This document states that "National Spatial Data Infrastructure (NSDI) means the technology, policies, standards, and human resources necessary to acquire, process, store, distribute, and improve the utilization of geospatial data". It also relates NSDI as "National Geospatial Data Clearinghouse" meaning a distributed network of geospatial data producers, managers, and users linked electronically. Since its formal inception, NSDI became one of the most powerful tools in decision-making process in many spheres of public sector domains.

For more than last two decades, applicability of NSDI has been grown-up widely because it enables sharing of spatial data in a fashionable manner which helps local, national and regional level users to achieve their targets by providing easy access to spatial data, reducing data duplication as well as saving time and financial resources (Putra, Sekimoto and Shibasaki, 2019). From the beginning of its journey, a number of NSDI initiatives are being adopted globally with the expectation to augment national planning and to support sustainable development (Cada and Janecka, 2016). An NSDI can be considered as a framework of technology, standards, policy, and collaboration among different organizations ensuring the access, sharing, exchange, and use of spatial data at national level in a country (Putra, Sekimoto and Shibasaki, 2019). There exists evidence of NSDI contributing positive influence on the socio-economic and environmental aspects of a country (Crompvoets et al., 2007; Campagna and Craglia, 2012).

Since the initial development, many developed and developing countries have adopted NSDI. Although, Bangladesh cannot be considered as an early adopter of NSDI but still it has made some remarkable progress in the establishment of NSDI in the

\footnotetext{
* Corresponding author
} 
country. The way, Bangladesh has mentionable success stories in using NSDI, still full-scale implementation foresees many challenges to be overcome to get desirable benefit of NSDI. This paper aims to present the development of NSDI in Bangladesh, its progress and way forward.

This paper is structured as follows. In Section 1, concept of NSDI has been introduced while Section 2 elaborately describes the development of NSDI in Bangladesh. Most importantly, the basis and roadmap of NSDI, current usage of geospatial data and vision for NSDI have been described. In Section 3, we have discussed the most favourable geospatial data platform including its components and rationale. Section 4 describes the current platform of Bangladesh NSDI and some applications about how present NSDI is facilitating different organizations. This paper ends with Section 5, where we have identified some challenges to implement full-fledged NSDI in Bangladesh.

\section{DEVELOPMENT OF NSDI IN BANGLADESH}

\subsection{The basis}

Government of Bangladesh formally announced the concept of "Digital Bangladesh" in 2009. Digital Bangladesh introduced EGovernance and service delivery to citizens through the use of information and communication technology (ICT). Digital Bangladesh is a vision for e-Government which aims to improve the efficiency and transparency of public administration and socio-economic growth through proper and wide use of ICT (Islam and Gronlund, 2011; Siddiquee, 2012; Hussain and Mostafa, 2016). By the policy maker it has been realised that NSDI is indispensable part and can be very instrumental to achieve Digital Bangladesh. It has also been recognised that proper utilization of NSDI for public and other facilities is indispensable for the formulation or release of various plan proposals and operation/maintenance of infrastructure when establishing digital government and upgrading citizen services and it is expected that this will contribute to economic growth and a transition to an information society supporting the achievement of sustainable development goals (SDGs).

Against this background, an international seminar on National Spatial Data Infrastructure (NSDI) was organised by Japan International Cooperation Agency (JICA) and Survey of Bangladesh (SoB) in 2016. IN this seminar, international keynote speakers from Japan, Indonesia and India shared their experience. The threadbare discussion in that seminar made participants understand about NSDI, it's necessities and way forward to establishment of Bangladesh NSDI.

In this seminar it has been discussed that, valuable mapping information as well as all geospatial information stored by different agencies need to be brought under a centralised platform. This will facilitate the relevant organisations and public users to have access this geospatial information for use in many sectors including urban/ rural planning, disaster management, public-service domain, infrastructures planning. This is also expected to facilitate people's participation in decision making contributing to socio-economic development of Bangladesh

Honourable Prime Minister Sheikh Hasina also attended the inauguration of this seminar as chief guest and she realised that development of NSDI is of paramount importance for the national development of Bangladesh. She also urged to form a national committee to develop the Bangladesh NSDI. In addition to this Honourable Prime minister also emphasized to prepare NSDI act. / Policy/ Rules in order to accelerate establishment of NSDI (JICA, 2016).

\subsection{Geospatial Data Usage In Bangladesh}

This history of using GIS and spatial data in Bangladesh dates back to the early 1990s when spatial data collection and storage system was initiated for Irrigation Support Project for Asia and the Near East (ISPAN) under the Bangladesh Flood Action Plan (FAP) project. The objective of the GIS set-up was to facilitate flood disaster mitigation in Bangladesh (CEGIS, 2009). However, successful implementation of this GIS set-up led to spreading the use of GIS technology among different government departments, national and international NGOs. Since then, use of GIS for national and local level development plans in Bangladesh is being used widely. Many organizations in Bangladesh have been using GIS for planning and decisionmaking process starting from local to regional to national to global levels. At present a significant number of organisations in Bangladesh have developed their own system to produce, update and disseminate geospatial data.

The organisations prominent in producing and using geospatial data are Survey of Bangladesh (SoB), Local Government Engineering Department (LGED), Bangladesh Water Development Board (BWDB), Department of Public Health Engineering (DPHE), Center for Environmental and Geographic Information Services (CEGIS), Water Resources Planning Organization (WARPO), Department of Land Records and Survey (DLRS), Soil Resources Development Institute (SRDI), Bangladesh Agricultural Research Council (BARC), Geological Survey of Bangladesh (GSB), Bangladesh Inland Water Transport Authority (BIWTA) and Institute of Water Modelling (IWM). These organisations produce spatial data of different types according to their needs and mandates. The spatial data produced by different organisations includes but not limited to Topographic map of different scales, Digital Elevation Model (DEM), Administrative boundaries of different levels, flood inundation and extent map, hazard map, lad use map, physical infrastructure map, agricultural land zoning and crop mapping, arsenic contamination map, soil resource map, facility location and distribution map, route map, transport network map etc.

Different organisation uses GIS as a decision making and planning tool. The application of spatial data in Bangladesh depends primarily on their rule of business and domains. Notable applications of using spatial data may be attributed to land use zoning and planning in urban area, environmental management, agricultural zoning, ecological mapping, infrastructure planning, rural development planning, disaster management, population mapping and poverty mapping etc. Now a days, there are also advancement in using GIS e.g WebGIS, location-based service and business analysis.

\subsection{Setting The Roadmap}

Before building NSDI, it was clearly recognized that only the establishment of the laws (for example, NSDI Act and Survey Act) and creating an appropriate system will not be sufficient for the utilization of NSDI by the relevant user group. In order to successfully implement and operate Bangladesh NSDI many technical, legal, administrative, and other issues needs to be solved. But, this will require much tame for resolving the 
interconnected problems and full operation of NSDI. Based on the objectives of establishment and operation of NSDI in Bangladesh, three successive periods are proposed: a) Preparation period, b) Infrastructure formation/Dissemination period and c) Operation period (Mid-term plan and long-term plan) (JICA, 2017). The objective and main actions in each period are presented in the Table 1 .

\begin{tabular}{|c|c|c|c|}
\hline \multicolumn{2}{|r|}{ Period } & Objective & Main Activities \\
\hline & 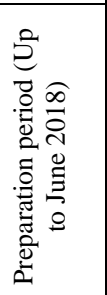 & 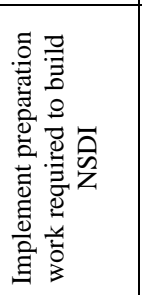 & $\begin{array}{l}\text { Building of Geo Portal website, Passage of } \\
\text { Survey Act, Promotion of NSDI Act, } \\
\text { Completion of digital topographic map, } \\
\text { Preparations for establishment of NSDI } \\
\text { Committee }\end{array}$ \\
\hline : & 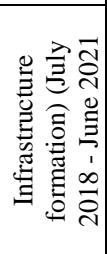 & 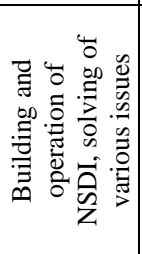 & $\begin{array}{l}\text { Building of NSDI platform, Passage of NSDI } \\
\text { Act, Establishment of NSDI Committee and } \\
\text { working group activities, Creation of primary } \\
\text { data and updating, / Updating and release of } \\
\text { digital topographic map for Dhaka City, } \\
\text { Review of base map updating technique, }\end{array}$ \\
\hline 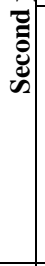 & 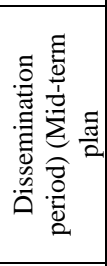 & 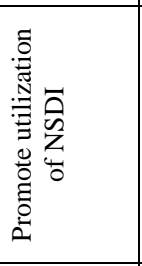 & $\begin{array}{l}\text { Periodic updating of base maps, } \\
\text { Development/updating of primary data with } \\
\text { high importance, Formulation of digital } \\
\text { topographic map development plan in other } \\
\text { local cities, Formulation and implementation of } \\
\text { promotion plan of geographic information } \\
\text { standards, Expansion/deployment of NSDI } \\
\text { platform, Operation and dissemination. }\end{array}$ \\
\hline | & 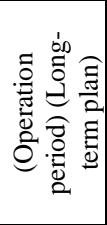 & 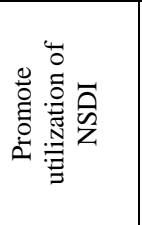 & $\begin{array}{l}\text { Examination of human resource development } \\
\text { methods including other ministries concerning } \\
\text { GIS, and implementation, Periodic updating of } \\
\text { base maps, Renewal of NSDI platform, } \\
\text { Dissemination of GIS into the work of } \\
\text { government agencies, Enhance map literacy of } \\
\text { citizens. }\end{array}$ \\
\hline
\end{tabular}

Table 1. Proposed development phases and activities (JICA, 2017)

\subsection{NSDI Committee}

The proposed NSDI Act is still under process to be approved by Prime Minister and after being approved it will be placed before parliament for final approval. This NSDI Act proposes a highlevel committee for the building and operation of NSDI in an efficient way. The proposed three-tires NSDI Committee are as follows:

a) National Committee

Headed by Prime Minister (Minister level committee) Headed by Secretary of MOD (Secretary level committee) Headed by Surveyor General of SOB (Department level committee)

The proposed three-tires committee are comprised of a) National Committee: Minister from each ministry, b) Technical Committee: Secretary from each ministry and agency and c) Executive Committee: Manager of each department. Accordingly, the working groups under the Executive Committee are supposed to review the various issues related to NSDI and will submit the recommended measure to Executive Committee. Among others most important two tasks are a) Technical issues related to NSDI and b) Operation/utilization issue of NSDI. Technical issues for geospatial information on NSDI are unification of administrative boundaries, coordinates conversion, unification of elevation standard, unification of geographic names, unification of address display format, unification of map symbols, data standardization, product specifications, metadata, format and other settings etc. whereas issues related to operation/utilization of NSDI are promoting utilization of NSDI to achieve greater economic development, developing data release policy, developing/updating, human resource development, building/expansion/operation of platform and other relevel works (JICA, 2017).

\subsection{Vision for Building Geoportal}

Multiple government agencies in Bangladesh have already established websites in order to release geospatial information to the general public. For example, the Bangladesh Computer Council (BCC) has already established and is operating a data sharing system called GeoDASH ${ }^{1}$ whose main objective was disaster information management. The GeoDASH system will be discussed in the later part. Flood Forecasting and Warning Centre (FFWC $)^{2}$ maintains a web-based system to predict and spatially visualize flood scenario to facilitate decision making and preparedness against flood disaster. Local Government Engineering Department (LGED) ${ }^{3}$ developed web-based platform for storage and sharing of spatial data. Although some other organisations are producing many spatial data, but they do not have web-based geoportal. In connection with building geoportal for NSDI, it was realised, that the overall cost required for building NSDI can be reduced by effectively utilizing the existing systems (avoiding redundant investments), enabling the system operation and maintenance. In consideration of the above facts, it was decided that NSDI would be built in a cooperative manner with the existing data sharing systems (JICA, 2017). NSDI Platform will distribute fundamental geospatial information that is the benchmark for locations, allow users to superimpose various geospatial information that they possess, and provide a mechanism that enables shared usage (sharing of geospatial information). An image of system usage is shown in Figure 1.

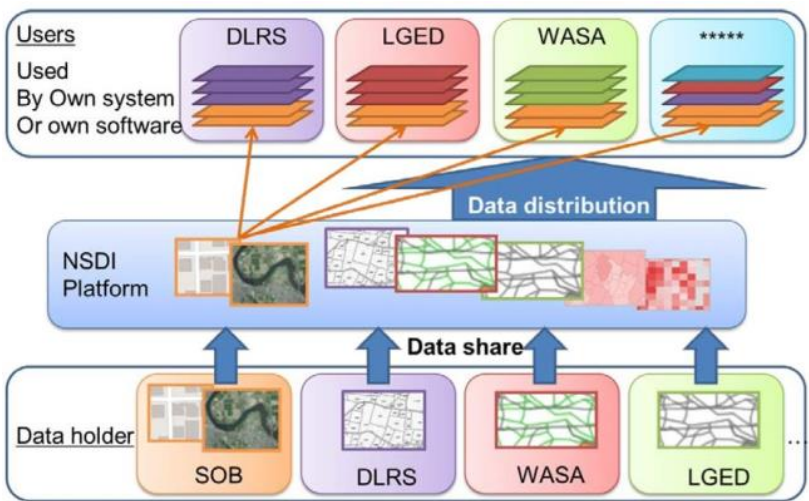

Figure 1. Image for NSDI platform system usage by related organizations (JICA, 2017)

Based on the functionality and openness of data sharing, decision was made that, the Basic Map Sharing System will be built by utilizing the GeoDASH platform. After the system is built, delivery settings will be made for the WMS Web Map Service and Web Map Tile Service so that the background maps

\footnotetext{
https://geodash.gov.bd/

${ }^{2}$ http://www.ffwc.gov.bd/

${ }^{3}$ http://gis.lged.gov.bd/
} 
can be referred to from other systems. It was envisioned that NSDI system will contribute to disaster-prevention planning, rebuilding support for disaster management, urban planning, rural planning, transportation planning and health and sanitation planning and so on. Figure 2 illustrates the spatial data and relevant application domains of NSDI.

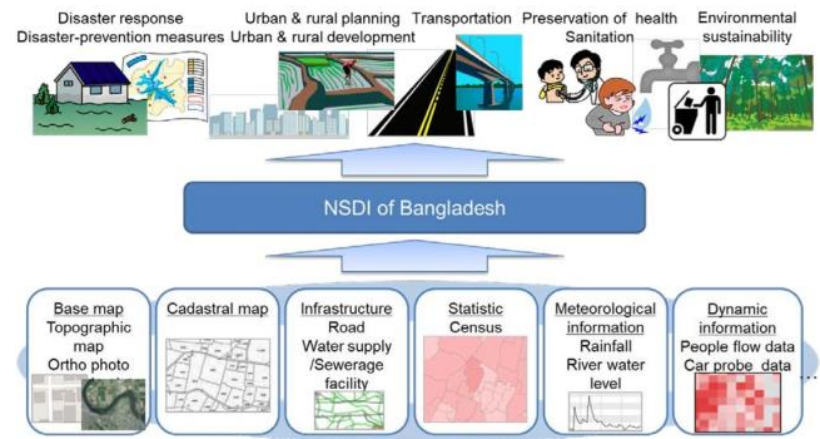

Figure 2. Spatial data and related application domains of proposed NSDI (JICA, 2017)

\subsection{NSDI Functions}

The functions that need to be provided to government institutions, private sector companies, including general users and academic institutions consist of Search, View, Delivery, Geo-processing and User Management. The functions that can be used by users depend upon user privileges and data limitations. Functions on the Data Holder side consist of functions concerning uploading of data to NSDI platform and data management. Proposed NSDI platform function configuration has been presented in the Figure 3.

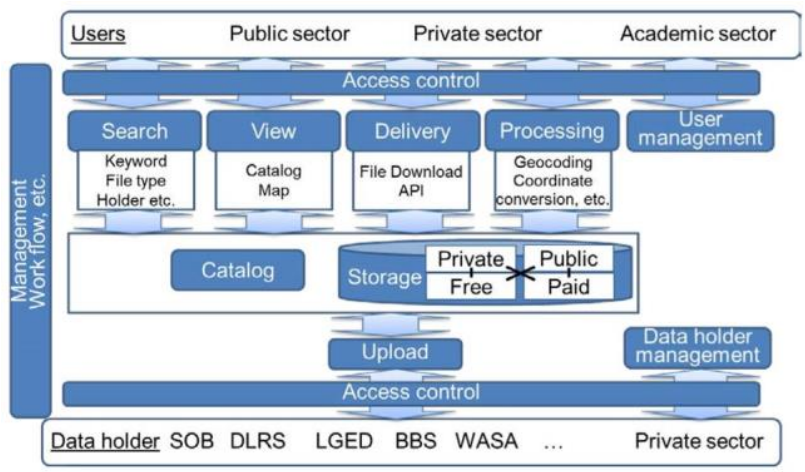

Figure 3. Proposed NSDI platform function configuration (JICA, 2017)

The main functions of the proposed NSDI platform are divided into data provision, data use and system management. Main activities under data provision will be registration, update and deleting of geospatial data. Organization involved in producing spatial data will have access to these activities in NSDI platform. User group will be able to search, query, analysis, and display of spatial data. People will also enjoy preparing, visualising and exporting/downloading the maps in the system. Figure 4 shows a sample of user created map in the NSDI platform showing the location of cyclone shelters in coastal zone of Bangladesh. System management is the administrative domain of the platform. Only system administrator will have access to this component. System administrator will control the overall platform and perform administrative activities including approval of user registration, spatial data checking and approval to upload, data modification and controlling the level of access among stakeholders.

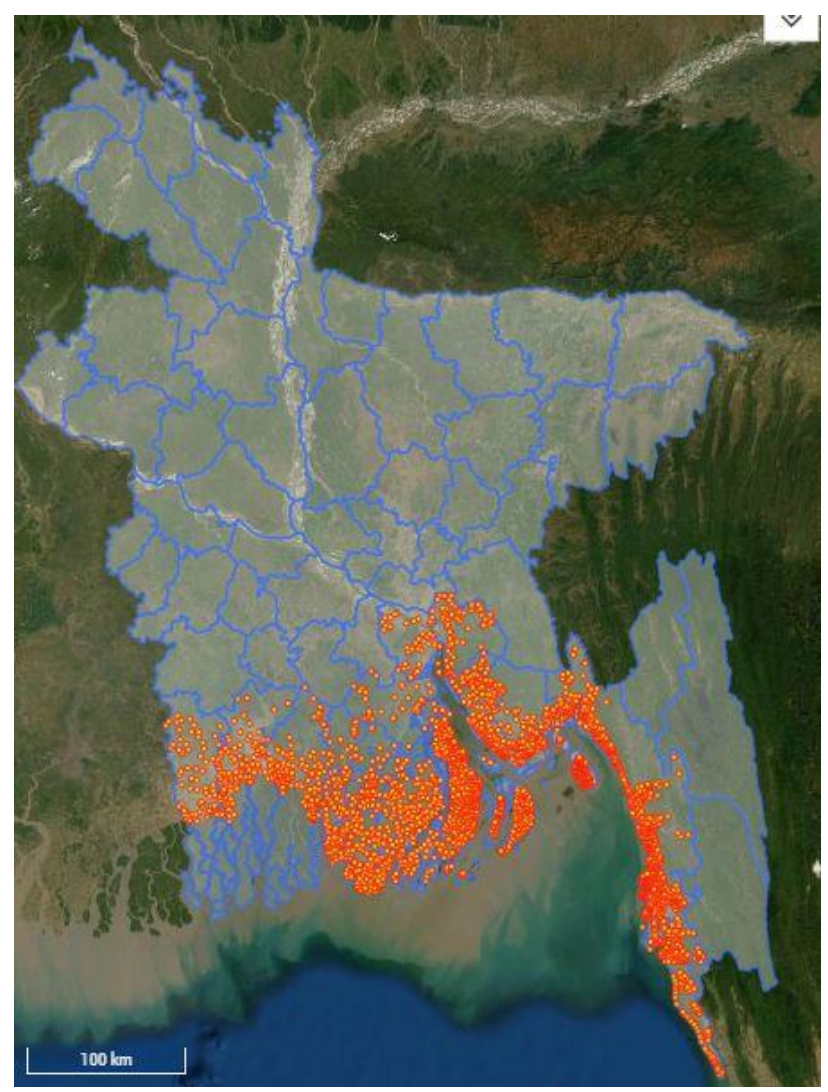

Figure 4. User created map showing location of cyclone shelters in coastal zone of Bangladesh.

\subsection{Formation of Bangladesh GIS Platform (BGISP)}

On $12^{\text {th }}$ May of 2011, a meeting of Economic and Social Council (ECOSOC) of UN was held in Geneva to form United Nations Committee of Experts on Global Geospatial Information Management (UNGGIM). This committee was formed with the objective to patronize its member state to integrate statistical data with geospatial data for sustainable development. This is the global level initiative to use geospatial data for helping sustainable development.

In Bangladesh, different organisations are engaged in GIS related activities but due to lack of coordination among those organisations, there are duplicate efforts and wastage of money and time. Hence, the country is deprived from expected benefits from the use of GIS. In connection with UNGGIM formed in Geneva, Bangladesh convinced that it is of paramount importance to coordinate among the different ministries/divisions, agency and private organizations to gather all the GIS actors in a single platform where they can share their activities and experiences among themselves which in return will reduce costs and wastage of time. Bangladesh GIS Platform (BGISP) $)^{4}$ is created to serve this purpose as per the provision of Section No. 6 of Statistical Act, 2013. A government order has been issued to create the platform headed by Additional Secretary, Statistics and Informatics Division, Ministry of

\footnotetext{
${ }^{4} \mathrm{http}: / /$ www.gis.gov.bd/
} 
Planning on 30th June, 2016 (BGISP, 2016). Bangladesh GIS Platform is the central GIS repository which accumulates all the GIS data from all GIS agencies of Bangladesh in a single platform as per the provision of Section No. 6 of Statistical Act, 2013. A government order has been issued to create the platform headed by Additional Secretary, Statistics and Informatics Division, Ministry of Planning on 30th June 2016 incorporating 36 (Thirty Six) members from 34 (Thirty Four) organizations. This committee will also maintain international liaison regarding geospatial data sharing for sustainable development.

\section{GEOSPATIAL OPEN DATA SHARING PLATFORM (GEODASH)}

\subsection{Rationale for Building GeoDASH}

The Bangladesh Urban Earthquake Resilience Project (BUERP) was started in 2011 with the support from the World Bank, the Global Facility for Disaster Reduction and Recovery (GFDRR) and Earthquakes and Megacities Initiative (EMI). The project aimed to establish the required institutional structure, knowledge base and data for multi-stakeholder engagement in order to address vulnerabilities from earthquakes in Bangladesh. BUERP had collected and generated new geospatial information and knowledge on earthquake hazard, vulnerability and risk assessment (HVRA). It was realised that for effective use of this information, an open platform is needed for sharing geospatial data with stakeholders and organizations involved in disaster risk reduction. In BUERP, there was a core component of establishing a Platform for existing earthquake hazard vulnerability data hosting, sharing and dissemination (World Bank and EMI, 2014). Throughout the undertaking of the BUERP, the stakeholders took a very active involvement in this component of the project and provided significant input as well guidance on both the opportunities as well as the challenges to make a data sharing platform a reality. The creation of a platform for data sharing was also ranked by these stakeholders as a high priority. After several seminars, workshop and threadbare discussion there was a consensus for building Geospatial Data Sharing Platform (GeoDASH)

The ultimate vision for a fully implemented GeoDASH Platform was that all relevant stakeholders have access to geospatial data required to conduct spatial analysis of disaster impact and support decision making in all phases of Disaster Risk Reduction Management (DRRM): mitigation, preparedness, response and recovery. Starting with urban earthquake risk management, the GeoDASH Platform will provide integrated data storage, data retrieval capabilities, viewing and mapping capabilities all in one nodal data sharing application. The vision was for GeoDASH to be scalable through linking of additional organizations in a "federation" of data sharing nodes, and extensible through incorporation of more data and features; for example from earthquake related information to other types of hazards and risk, or from Dhakaspecific database to other cities in Bangladesh or a nationallevel database (World Bank and EMI, 2014).

\subsection{Pillars of GeoDASH}

It was envisioned that Geospatial Open Data Sharing Platform (GeoDASH) will be founded as three inter-related elements. It was also envisioned that the Data Sharing platform should be founded in accordance with the existing legal directives, data sharing through ICT and information sharing/dissemination protocol related to disaster management of Bangladesh. This rational serves as the basis for anchoring among the stakeholders and ensures that GoDASH to be built upon and is linked to the existing ICT structure and legal mandate.

GeoDASH is grounded by three interrelated components. These are organizational components, technical components and capacity building components as presented in the Figure 5 . Technical component is the central pillar and is considered as the backbone of the open data sharing platform. This component is dedicated to serve the technical aspects of the platform supplying the data, applications and infrastructure required to operationalize the system. By its nature, the organizational components set the responsibilities and functions among the stakeholder institutions in order to ensure successful production and data sharing protocol. This acts as a coordinating agent in the overall system. The capacity building is responsible to strengthen the technical and capacity and other ability of the stakeholder institution to ensure efficient production and sharing of spatial data in a standard metadata form as agreed upon. This component will organise training for the capacity building of the human resources. All the components are described below.

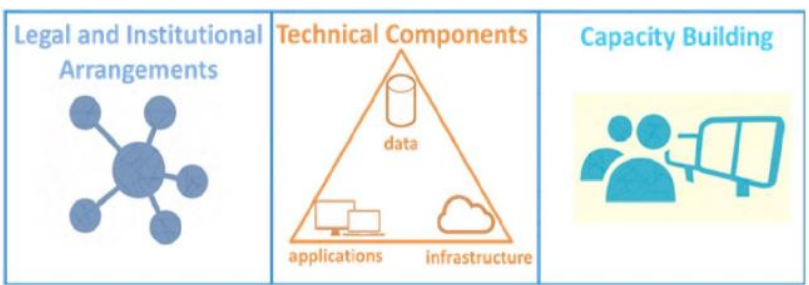

Figure 5. Three key elements (pillars) of the Geospatial Data Sharing Platform (World Bank and EMI, 2014)

3.2.1 Technical Component: The technical component of the GeoDASH Platform is composed of three key elements which includes a) Geospatial data, b) Software applications, and c) Data hosting infrastructure. These three elements have been considered as integrated geospatial suite which will serve as the core components of overall GeoDASH system (World Bank and EMI, 2014).

In the first step, Geospatial data incorporated in the initial configuration of GeoDASH was based on the primary geospatial data created as part of the HVRA (Hazard, Vulnerability, Risk Assessment) Component of the BUERP. Under BUERP, different organisations like Dhaka City Corporation (DCC), Rajdhani Unnayan Kartripakkha (RAJUK), Bangladesh Bureau of Statistics (BBS) and Department of Disaster Management (DDM) were involved. These organisations created many geospatial data. Those data were compiled in a common metadata standard. Initially GeoDASH was based on those data only.

There exists numerous software platform to be used spatial data sharing hub. In this it was thought that this system will use such software applications that can ease performing geospatial data sharing, analysis and visualisation. It was also considered that this system will provide such access to use group that will enable downloading, customizing to meet the verities of day-today needs.

GeoNode was proposed in the Roadmap as a web-based software application for the sharing of geospatial. GeoNode is a web-based application and platform for developing geospatial information systems (GIS) and for deploying spatial data 
infrastructures (SDI). It is a web framework, based on an open source software technology stack, which enables organizations to build and deploy geospatial content management systems (GeoCMS), public spatial data infrastructures (SDI) (Kralidis, 2009) and open geospatial data catalogues. The Global Facility for Disaster Reduction and Recovery (GFDRR) started GeoNode development in 2009 and since then the project was joined by a large number of organizations, universities and research centers. GeoNode is a web application which allows users to share geospatial datasets and combine them in thematic web maps. All the datasets are accessible from clients using Open Geospatial Consortium (OGC) standards such as Web Map Service (WMS), Web Feature Service (WFS), Web Coverage Service (WCS), Catalog Service for the Web (CSW). GeoNode's data management tools allow for integrated creation of data, metadata, and map visualizations. Each dataset in the system can be shared publicly or restricted to allow access to only specific users.

In the past years, there success history of using and deploying Geonode as a geospatial data sharing platform in disaster risk management. Haiti Data ${ }^{5}$ can be mentioned as the instance of GeoNode deployment for Haiti emergency activities. Caribbean Geonode Platform is another example of Geonode deployment in climate risk management. Geonode is adorned with mature and stable open-source software projects which allow consistent and easy-to-use interface even for non-specialized users in sharing geospatial data and preparing interactive maps.

Physical and logical components of IT have been included in data hosting infrastructure that allow to be leveraged by multiple users. The physical and logical components includes but not limited to Data centers and data repositories, including the cloud; Networks, including Internet and intranets; Host platforms for data, services, and applications, including portals and services; Network routing services and Access control and security (World Bank and EMI, 2014).

In the BUERP project, a version of Geonode was first installed by EMI with the help of the Amazon AWS cloud service in order to test the permormance of GeoNode as it was recommended by world bank technical staff. Before installing GeoNode in BUERP, various options were tested through pilot project of GeoNode in local server as well as on the cloud. This allowed the connected institutions to manage and maintain the data from their own location.

3.2.2 Organisational Component: Organisational component is another one in the GeoDASH system. With this component, all the organisations, institutions involved in producing and sharing geospatial data are engaged. This component defines the role, functions, and activities to be done by different organisations in order to facilitate spatial data production and sharing in an effective and coordinated manner. Primarily it was decided that the system will operate in Dhaka and then beyond. Three key aspects of this element are considered here and include: 1) the roles and functions of the respective institutions; 2) coordination mechanisms and models for data sharing; and 3) protocols and processes by which to manage geospatial assets of the Geospatial Data Sharing Platform (World Bank and EMI, 2014).
3.2.3 Capacity Building Component: The main purpose of the capacity building component is to strengthen the ICT related capacity among the staffs of related organisation so that they can easily produce, modify, and share the geospatial data in a common standard. In order to assess the capacity building requirements, the required data was collected from different stakeholders through inventory checklist and guided interviews. This was done to identify the gaps between the optimized allocation and integration of the inputs in terms infrastructure and human capacity needed to make the GIS and ICT system to be ready for use in disaster management system. Then series of trainings, workshops, seminars were conducted to ensure that the Data Sharing Platform is used and maintained in an appropriate way. From the capacity assessment, it was revealed that different organisations required different types of capacity building such as technical capacity, infrastructure, manpower etc. Depending on the existing status of GIS capacity in the respective organization, capacity development plan was modified to fit the varied needs (World Bank and EMI, 2014).

\section{GEODASH BASED NSDI IN BANGLADESH}

Despite the usage of geospatial data by many organizations in Bangladesh for years, there was evidence of SDI just 4-5 years ago. In this regard, World Bank has taken a premier initiative to establish Geospatial Data Sharing (GeoDASH) Platform. This platform is considered as a complete SDI. GeoDASH is a webbased geoportal empowering government, academia, private enterprise, and the public to securely host, manage, share, visualize and analyse geospatial data. Developed on open source software GeoNode, it facilitates collecting, storing, and sharing geospatial data. Considering the long-term sustainability, maintenance and technical competencies, in December 2015, the ownership of Geospatial Data Sharing Platform GeoDASH) has been transferred to the Bangladesh Computer Council (BCC), Government of Bangladesh (GoB). $\mathrm{BCC}$ hosted the GeoDASH at its own data center with web GeoDASH.gov.bd (Bari, 2018).

Since its initiation, establishment of Bangladesh NSDI has achieved a significant progress. Survey of Bangladesh (SOB) is acting as leader to develop NSDI with support from JICA. BCC is also providing technical support to SoB. In early of 2018, it was decided that GeoDASH will be used as the platform for Bangladesh NSDI. A snapshot view of GeoDASH has been presented in Figure 6. For making the GeoDASH to be robust, some customizations/ modifications have been done on GeoDASH platform. Many organisations are collaborating with SoB to be connected with GeoDASH platform for data sharing purpose. However, still, many organisations are in left behind with this platform. But it is expected that all relevant organisations will be involved in coming days. After completion of the pilot work, output of the NSDI progress will be presented in some national conference. Through this conference feedback will be collected from the stakeholders. After incorporating the comments from stakeholders, the final version of NSDI will go for live. Salient features of Bangladesh NSDI are as follows (Bari, 2018):

\footnotetext{
$\checkmark \quad$ Unified projection system

$\checkmark \quad$ Provision to setup custom base map

$\checkmark \quad$ Data management module

$\checkmark \quad$ Administration and user categories

$\checkmark$ Access control

$\checkmark \quad$ Web mapping service
}

\footnotetext{
${ }^{5}$ http://www.haitidata.org/
} 
$\checkmark \quad$ Cross layer buffer search

$\checkmark$ Analytics

$\checkmark$ Data Security

$\checkmark \quad$ Scalable platform to handle large data

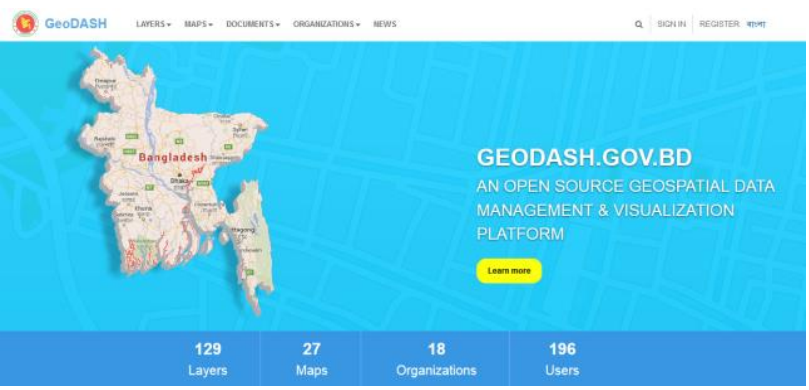

Figure 6. Snapshot of GeoDASH Platform

\subsection{Some applications of GeoDASH}

Since its inception, GeoDASH is being used widely by many sectors in Bangladesh. For example, Directorate of Primary Education of Bangladesh has assessed 35,000 schools considering infrastructure, water and sanitation facilities, access to roads, and overall capacity during natural disasters using the geospatial data in GeoDASH. Vulnerable road communication due to flood was evaluated using geospatial data. For example, locations of road network data and flood inundation depth were overlaid to identify vulnerable road communications due to flood. Such map helps the policy maker to understand how the road infrastructure will exposed due to flood, hence assisting quick decision making and planning. Many types of exposure analysis are being conducted using geospatial data.

Likewise, Department of Disaster Management (DDM) has shared the cyclone-induced storm surge inundation depth layer which could be used for creating cyclone risk maps with vulnerable infrastructure (e.g., primary schools). These risk mapping efforts will inform larger investments in resilience and help to invest in cyclone shelters cum primary schools with access to roads.

LGED is using this geospatial data for its planning and construction of infrastructure in local level urban and rural areas of Bangladesh. Figure 7 shows a sample flood exposure analysis of urban infrastructure. Spatial data (infrastructure) used in this analysis has been produced by LGED in connection with the Upazila Town Infrastructure Development Project (UTIDP).

Bangladesh Forest Department is a government agency responsible for the protection and maintenance of forests and wildlife in Bangladesh. This agency has been producing many spatial data according to their needs. They have developed Bangladesh Forest Information System (BFIS) based on GeoDASH platform.

Bangladesh Government has developed a web-based SDG tracking system ${ }^{6}$ to monitor and spatially visualize SDG indicators over the country. Bangladesh Bureau of Educational Information \& Statistics (BANBEIS) has developed a geoportal $^{7}$ for the management and decision support system for the education information across the country.

\footnotetext{
${ }^{6} \mathrm{http}: / /$ www.sdg.gov.bd/

${ }^{7}$ http://www.banbeis.gov.bd/
}

Apart from these, many organisations like Dhaka North and South City Corporations (DNCC and DSCC), Capital Development Authority (RAJUK), Bangladesh Water Development Board (BWDB), Flood Forecasting and Warning Center (FFWC), Rural Electrification Board of Bangladesh (REB), Dhaka Water and Sewerage Authority (DWASA), have already started hosting their spatial data and information and map in the GeoDASH for sharing, improved analysis, decision making and planning to support the achievement of SDGs.

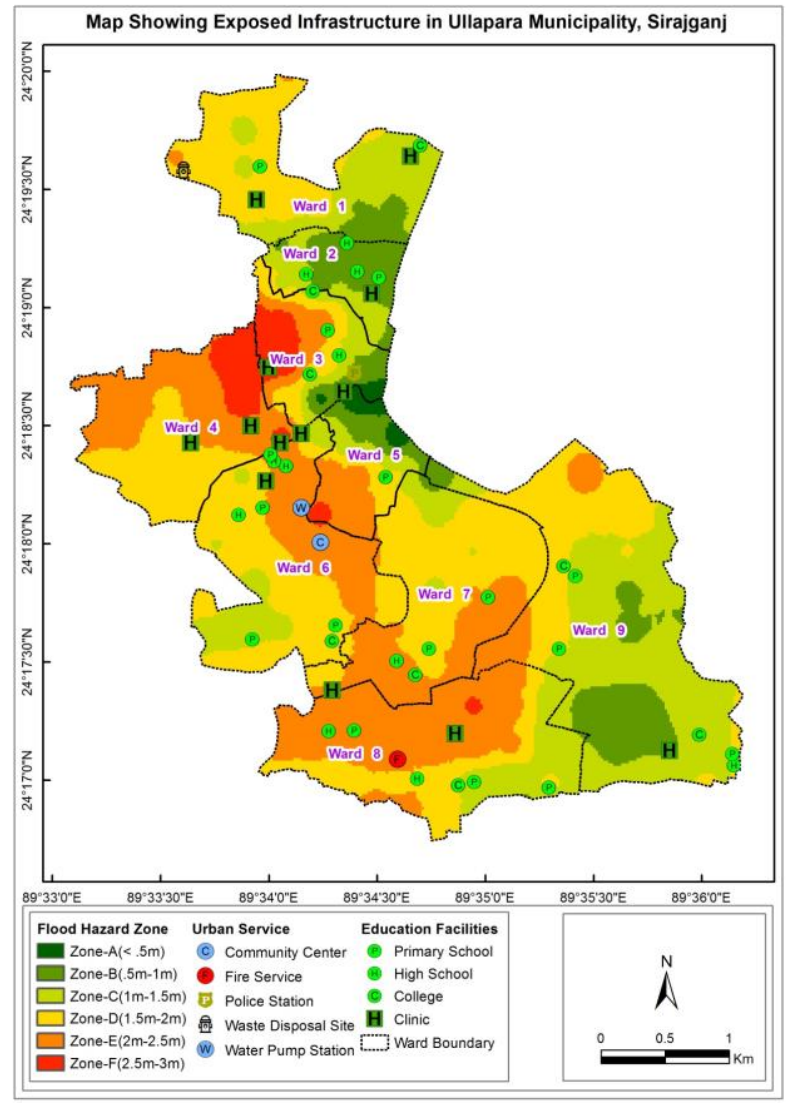

Figure 7. A sample of flood exposure analysis of infrastructure based on geospatial data

\section{WAY FORWARD/CONCLUSION}

Over last two decades many countries have developed their own NSDI system and getting benefits from them for decision making and need based planning with priority to support the achieve the SDGs. NSDI is helping in many ways in many sectors including urban planning, land use planning, agricultural land zoning, disaster management. Environmental monitoring etc. In line with the global progress Bangladesh is also committed to develop advanced level NSDI for country's planning in many sectors. However, full-fledged development of NSDI is still under process in Bangladesh. As described in different sections in this paper, many organisations in Bangladesh are producing and using geospatial data for their own purpose. Some of them have also developed their own geoportal for their own mandates. Now this is time develop centralised NSDI for greater benefit. Till now Bangladesh has made significant progress of developing NSDI. JICA, with its previous experience in Japan, is helping to develop a standardised NSDI in Bangladesh. At present GeoDASH platform, with some modification, is being used as NSDI 
platform in Bangladesh with support from JICA. In line with the prevailing policy, GeoDASH is expected to act as an anchor for geospatial data sharing among in all relevant government agencies in Bangladesh. It will encourage greater openness of non-sensitive data to all.

Fully operational NSDI has a great significance of achieving SDGs of developing country like Bangladesh. Since, reliable and timely accessible data is required to measure the progress towards SDGs NSDI can play a vital role in order to achieve SDGs in Bangladesh. Implementation of Bangladesh NSDI will face several challenges also. Major problems concerning the data compatibility. Many organisations in Bangladesh are using different projection systems, there are also lack of standard and lack of standard quality guidelines. Most of the organisations do not have any metadata standard. There is no standard guideline to share the relevant data during emergency period. Another challenge is the sustainability and security of the platform. Apart from these, coordination among different organisation is also challenging in Bangladesh. NSDI Act is still under process in Bangladesh. If all the challenges are overcome, it is expected that after being approved the NSDI Act, the development of Bangladesh NSDI will be straightforward and functional creating real value for the people, government, and SDGs achievement.

\section{REFERENCES}

Bari, T. (2018) 'NSDI Establishment for Digital Bangladesh', Computer Jagat, pp. 44-45. Available at: http://comjagat.com/archive/articleimage/How_facebook\%60s_ 50m_user_was_hacked_Nov._18(Q)_.pdf.

BGISP (2016) Bangladesh GIS Platform. Available at: http://www.gis.gov.bd/en/index.php (Accessed: 3 February 2020).

Cada, V. and Janecka, K. (2016) 'The strategy for the development of the infrastructure for spatial information in the Czech Republic', ISPRS International Journal of GeoInformation, 5(3). doi: 10.3390/ijgi5030033.

Campagna, M. and Craglia, M. (2012) 'The socioeconomic impact of the spatial data infrastructure of Lombardy', Environment and Planning B: Planning and Design, 39(6), pp. 1069-1083. doi: 10.1068/b38006.

CEGIS (2009) Compatibility of GIS Data and Development of Guidelines for Metadata, Data Sharing Protocol and National GIS Data Policy.

Clinton, W. (1994) Coordinating geographic data acquisition and access: the National Spatial Data Infrastructure. Executive Order 12906, Federal Register 59, Washington, DC, April 1994., Presidential Documents.

Crompvoets, J. et al. (2007) 'Worldwide impact assessment of spatial data clearinghouses', URISA Journal, 19(1), pp. 23-32.

Dale, P. and Allan, A. L. (2004) 'Developing Spatial Data Infrastructures - from concept to reality', Survey Review, 37(292), pp. 498-499. doi: 10.1179/sre.2004.37.292.498.

Hussain, F. and Mostafa, M. (2016) 'Digital contradictions in Bangladesh: encouragement and deterrence of citizen engagement via ICTs', Information Technologies \& International Development, 12(2), pp. 47-61.

Islam, M. S. and Gronlund, A. (2011) 'Digital Bangladesh - A change we can believe in?', in Lecture Notes in Computer Science (including subseries Lecture Notes in Artificial Intelligence and Lecture Notes in Bioinformatics), pp. 107-121. doi: 10.1007/978-3-642-22961-9_9.

JICA (2016) International seminar on National Spatial Data Infrastructure (NSDI) for Bangladesh. Available at: https://www.jica.go.jp/bangladesh/english/office/topics/160602. html (Accessed: 3 February 2020).

JICA (2017) Project for strengthen the capacity on advanced mapping of SOB for building digital Bangladesh: making the roadmap to establishing NSDI: final report. Available at: http://open_jicareport.jica.go.jp/548/548/548_101_12301982.ht $\mathrm{ml}$.

Kralidis, A. T. (2009) 'Geospatial Web Services: The Evolution of Geospatial Data Infrastructure', in The Geospatial Web, pp. 223-228. doi: 10.1007/978-1-84628-827-2_22.

Nogueras-Iso, J., Zarazaga-Soria, F. J. and Muro-Medrano, P. R. (2005) Geographic information metadata for spatial data infrastructures: Resources, interoperability and information retrieval, Geographic Information Metadata for Spatial Data Infrastructures: Resources, Interoperability and Information Retrieval. doi: 10.1007/3-540-27508-8.

Pashova, L. and Bandrova, T. (2017) 'A brief overview of current status of European spatial data infrastructures - relevant developments and perspectives for Bulgaria', Geo-Spatial Information Science, 20(2), pp. 97-108. doi: 10.1080/10095020.2017.1323524.

Putra, T. Y. D., Sekimoto, Y. and Shibasaki, R. (2019) 'Toward the evolution of national spatial data infrastructure development in Indonesia', ISPRS International Journal of Geo-Information, 8(6). doi: 10.3390/ijgi8060263.

Siddiquee, N. A. (2012) 'E-government and transformation of governance and service delivery in Bangladesh: A developing country perspective', in ACM International Conference Proceeding Series, pp. 271-278. doi: $10.1145 / 2463728.2463782$.

World Bank and EMI (2014) Road Map for Disaster Data Sharing Platform: Bangladesh Urban Earthquake Resilience Project. Available at: https://emi-megacities.org/wpcontent/uploads/2015/01/Data-Sharing-PlatformRoadmap_FINAL.pdf. 